novel lists of overlapping or functionally related genes through statistical or bioinformatic analysis.

With the collapse of R\&D in mental health by pharmaceutical companies, convergent/integrative 'omics' approach represents a unique opportunity for the scientific community to mine existing datasets as well as data from experimental and clinical models, to prioritize targets for the psychotropic medications of the future.

\section{FUNDING AND DISCLOSURE}

AC and CMP are supported by the UK National Institute for Health Research (NIHR) Biomedical Research Centre at the South London and Maudsley NHS Foundation Trust and King's College London; and by the UK Medical Research Council, Grants MR/ L014815/1 and MR/J002739/1. CMP has received research funding from pharmaceutical companies interested in the development of new antidepressants, such as Johnson \& Johnson and Eleusis, but the work described in this paper is not related to this funding. AC does not have any conflict of interest.

\section{Annamaria Cattaneo ${ }^{1,2}$ and}

\section{Carmine M Pariante ${ }^{1,2}$}

${ }^{1}$ Stress, Psychiatry and Immunology Laboratory,

Department of Psychological Medicine, Institute of

Psychiatry, Psychology \& Neuroscience, King's

College, London, UK; ${ }^{2}$ Biological Psychiatry Unit,

IRCCS Fatebenefratelli S. Giovanni di Dio, Brescia,

Italy

E-mail: carmine.pariante@kcl.ac.uk

Anacker C, Cattaneo A, Luoni A, Musaelyan K, Zunszain PA, Milanesi $E$ et al (2013). Glucocorticoid-related molecular signaling pathways regulating hippocampal neurogenesis. Neuropsychopharmacology 38: 872-883.

Ayalew M, Le-Niculescu H, Levey DF, Jain N, Changala B, Patel SD et al (2012). Convergent functional genomics of schizophrenia: from comprehensive understanding to genetic risk prediction. Mol Psychiatry 17: 887-905.

Luoni A, Massart R, Nieratschker V, Nemoda Z, Blasi G, Gilles $M$ et al (2016). Ankyrin-3 as a molecular marker of early-life stress and vulnerability to psychiatric disorders. Transl Psychiatry 6: e943.

Malki K, Du Rietz E, Crusio WE, Pain O, PayaCano J, Karadaghi RL et al (2016). Transcriptome analysis of genes and gene networks involved in aggressive behavior in mouse and zebrafish. Am J Med Genet B Neuropsychiatr Genet 171: 827-838

Niculescu AB 3rd, Segal DS, Kuczenski R, Barrett T, Hauger RL, Kelsoe JR (2000). Identifying a series of

candidate genes for mania and psychosis: a convergent functional genomics approach. Physiol Genomics 4: 83-91.

Patel SD, Le-Niculescu H, Koller DL, Green SD, Lahiri DK, McMahon FJ et al (2010). Coming to grips with complex disorders: genetic risk prediction in bipolar disorder using panels of genes identified through convergent functional genomics. Am J Med Genet B Neuropsychiatr Genet 153B: 850-877.

\section{(i)}

This work is licensed under a Creative Commons Attribution 4.0 International License. The images or other third party material in this article are included in the article's Creative Commons license, unless indicated otherwise in the credit line; if the material is not included under the Creative Commons license, users will need to obtain permission from the license holder to reproduce the material. To view a copy of this license, visit http:// creativecommons.org/licenses/by/4.0/

(C) The Author(s) 2018

Neuropsychopharmacology Reviews (2018) 43, 227-228. doi:I 0.1 038/npp.2017.221

\section{A Translational Model to Assess Sign-Tracking and Goal-Tracking Behavior in Children}

Cues or stimuli in the environment can guide behavior in adaptive ways, bringing one in close proximity to valuable resources (for example, food). For some individuals, however, environmental stimuli may acquire inordinate control over behavior and elicit maladaptive tendencies or intrusive thoughts. Thus, the way an individual responds to cues in the environment may be a key determinant of psychopathology. For example, in addiction, relapse is most often triggered by exposure to stimuli (for example, paraphernalia or places) previously associated with the drug-taking experience, and people suffering from posttraumatic stress disorder (PTSD) experience extreme anxiety or flashbacks upon exposure to stimuli reminiscent of a traumatic event. Furthermore, in patients with schizophrenia, psychosis is believed to result from aberrant attribution of motivational salience to environmental stimuli (Kapur, 2003). Such stimuli are able to elicit complex emotional and motivational states via Pavlovian learning, and in recent years we have come to rely on an animal model to better understand these processes (for review see Robinson et al, 2014).

When exposed to a Pavlovian conditioning paradigm wherein the presentation of a lever (conditioned stimulus, CS) is followed by delivery of a food reward (unconditioned stimulus, US), some rats, termed 'goal-trackers' (GT), attribute predictive value to the lever-cue and go to the location of food delivery upon cue presentation. Others, termed 'signtrackers' (ST), also attribute incentive salience to the lever-cue, as evidenced by their approach towards the cue and the ability of the cue alone to act as a reinforcer (for review see Robinson et al, 2014). That is, for ST the reward cue attains excessive incentive motivational value and gains inordinate control, leading to maladaptive behaviors. Indeed, relative to GT, ST have also been shown to be more impulsive, more likely to exhibit cue-induced relapse to drug-seeking behavior after relatively little drug exposure, and more susceptible to abnormal fear responses upon exposure to aversive stimuli (for review see Robinson et al, 2014). Thus, examining the translational relevance of the sign-tracker/goal-tracker model may prove critical to our understanding of a number of cue-motivated psychopathologies, including impulse control disorders, addiction and post-traumatic stress disorder.

To-date, little research has directly examined sign- and goal-tracking behavior in humans (Garofalo and di Pellegrino, 2015), and, to our knowledge, none with children. Due to the delayed development of the prefrontal cortex (Casey et al, 2000), children may be more likely to exhibit signtracking behavior. Indeed, the lack of cortical control and associated attentional deficits and impulsive behavior 


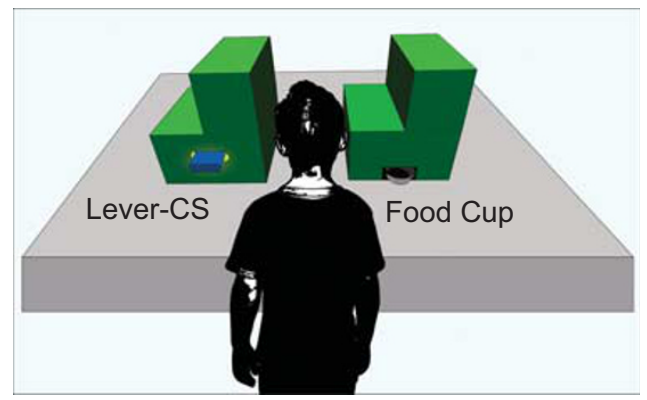

Figure 1. Pavlovian conditioning apparatus and resultant behavior. The Pavlovian conditioning apparatus consists of two child-friendly plastic Lego boxes. The CS box (left) contains a lever, which illuminates and extends from the box. The US box (right) contains a small metal tray into which candy is dispensed. The boxes are powered using an Arduino program controlled by a researcher on a laptop using MATLAB. For each trial, the lever-CS illuminates and extends for $8 \mathrm{~s}$, then darkens and retracts back into the box. Immediately upon CS retraction, the US box dispenses one piece of candy. Subjects are exposed to 4 blocks of 10 trials each. Following each trial is an intertrial interval (ITI) period, lasting 8, 16, 24 o $32 \mathrm{~s}$ (randomly chosen). The number of contacts with the lever-CS and US food cup, and the latency with which these occur during CS presentation are all recorded by the MATLAB program. Responses during the ITI are also recorded. Upon completion of each block the children are given a 45-second break.

evident in children is akin to that characteristic of sign-trackers in the animal literature (for review see Flagel and Robinson, 2017; see also Koshy Cherian et al, 2017). Capturing individual variation in cue-motivated behaviors in children may therefore provide a means to identify risk profiles for psychopathology early in life and thus offer earlier opportunities for intervention. In this regard, we have developed a novel apparatus to investigate signtracking and goal-tracking behaviors in children. The Pavlovian conditioning paradigm that we utilize is similar to that used in rodents (Figure 1) and consists of paired presentations of a lever (CS) with the delivery of candy (US). As in the animal paradigm, the children are allowed to freely move and manipulate the apparatus, and interaction with the lever-CS and candy tray are recorded. Using this paradigm, we have been able to observe both sign- and goal-tracking behavior (data not shown; to be published in a full-length manuscript). Ongoing studies are optimizing the behavioral output measures being assessed and examining the relationship between the propensity to exhibit a sign- or goal-tracking response and the development of psychopathology, including substance abuse and overeating. It is hoped that this translational model will prove invaluable for parsing the myriad of factors (for example, developmental, genetic, environmental, neurobiological) that render an individual more susceptible to cue-motivated psychopathologies and lead to novel therapeutic interventions.

\section{FUNDING AND DISCLOSURE}

This work was supported by funds from the Department of Psychology at the University of Michigan (MAJ and ANG) and funds from the Department of Psychiatry at The University of Michigan (SBF). The authors declare no conflict of interest.

\section{ACKNOWLEDGMENTS}

We thank Marc Bradshaw for building the testing apparatus and Chris Broussard and Igor Belopolsky for assistance with the computer programming. In addition, we would like to thank Dr. Paolo Campus for generating the schematic in Figure 1.

Michelle A Joyner ${ }^{1}$, Ashley N Gearhardt ${ }^{1}$ and Shelly B Flagel ${ }^{2,3}$

${ }^{1}$ Department of Psychology, University of Michigan, Ann Arbor, MI, USA; ${ }^{2}$ Department of Psychiatry, University of Michigan, Ann Arbor, MI, USA;

${ }^{3}$ Molecular and Behavioral Neuroscience Institute, University of Michigan, Ann Arbor, MI, USA

E-mail: sflagel@umich.edu

Casey BJ, Giedd JN, Thomas KM (2000). Structura and functional brain development and its relation to cognitive development. Biol Psychol 54: 241-257.

Flagel SB, Robinson TE (2017). Neurobiological basis of individual variation in stimulus-reward learning. Curr Opin Behav Sci 13: 178-185.

Garofalo S, di Pellegrino G (2015). Individual differences in the influence of task-irrelevant Pavlovian cues on human behavior. Front Behav Neurosci 9: 163.

Kapur S (2003). Psychosis as a state of aberrant salience: a framework linking biology, phenomenology and pharmacology in schizophrenia. $A m \mathrm{~J}$ Psychiatry 160: 13-23.

Koshy Cherian A, Kucinski A, Pitchers K, YEgla B, Parikh V, Kim Y et al (2017). Unresponsive choline transporter as a trait neuromarker and a causal mediator of bottom-up attentional biases. J Neurosci 37: 2947-2959.

Robinson TE, Yager LM, Cogan ES, Saunders BT (2014). On the motivational properties of reward cues: individual differences. Neuropharmacology 76(Part B): 450-459.

Neuropsychopharmacology Reviews (2018) 43, 228-229. doi: 10.1 038/npp.2017.196

\section{The Role of \\ Mitochondrial Glutamate Metabolism in Cognitive Development and Disease}

Glutamate metabolism serves a critical role in a variety of processes that regulate cognition, including excitatory synaptic transmission, energetics, and biosynthesis. We and others have recently identified a new genetic disorder of human cognitive development that involves a mitochondrial enzyme with a role in glutamate metabolism (Celis et al, 2015; Lobo-Prada et al, 2017; Ouyang et al, 2016). Investigation of this new neurogenetic disease promises valuable insight into the multiple functions of mitochondria and glutamate metabolism in brain development and cognition.

Through linkage mapping to chromosome 16 and high-throughput sequencing, mutations in a mitochondrial enzyme, glutamate pyruvate transaminase 2 (GPT2), have been identified in pedigrees affected by intellectual disability and postnatal microcephaly (Celis et al, 2015; LoboPrada et al, 2017; Ouyang et al, 2016). Also, a subset of patients has a progressive motor dysfunction, termed spastic paraplegia (Ouyang et al, 2016). 